Succesive approximation method for some linear boundary value problems for differential equations with a special type of argument deviation

A. Rontó and M. Rontó 


\title{
SUCCESSIVE APPROXIMATION METHOD FOR SOME LINEAR BOUNDARY VALUE PROBLEMS FOR DIFFERENTIAL EQUATIONS WITH A SPECIAL TYPE OF ARGUMENT DEVIATION
}

\author{
A. RONTÓ AND M. RONTÓ
}

Received 6 November, 2008

\begin{abstract}
We discuss successive approximation techniques for the investigation of solutions of some linear two-point boundary value problems for differential equations with special types of argument deviation.
\end{abstract}

2000 Mathematics Subject Classification: 34B15

Keywords: Functional-differential equation, special deviations of argument, linear boundary value problem, successive approximations

\section{INTRODUCTION}

In studies of various boundary value problems, it is often important to possess techniques based upon some types of successive approximations constructed explicitly in an analytic form. To this class of methods belongs, in particular, the approach suggested in [19-22] and originally aimed at the investigation of the periodic boundary value problem

$$
\begin{gathered}
x^{\prime}(t)=f(t, x(t)), \quad t \in[0, T], \\
x(0)=x(T),
\end{gathered}
$$

which corresponds to the problem on periodic solutions of systems of first order ordinary differential equations with non-linear non-autonomous time-periodic right-hand sides [22]. Appropriate versions of the method can be applied in many situations to systems of first or second order ordinary differential equations, integro-differential equations, equations with retarded argument, and other more general functionaldifferential equations under various boundary conditions (periodic, Cauchy-Nicolletti, multipoint, etc.; see, e.g., [10,23] for a bibliography). A survey of many papers devoted to the issues mentioned and published during the last three decades can be found in [11-17]. The approach indicated suggests one to replace the given boundary value problem by a family of Cauchy problems for a suitably perturbed differential 
system containing some artificially introduced parameter $z \in \mathbb{R}^{n}$ whose value is to be determined later. This idea is most transparent for the periodic boundary value problem (1.1), (1.2) considered in [19-22], in which case the parameter has the meaning of the initial value of the solution at zero and the corresponding Cauchy problem has the form

$$
\begin{aligned}
x^{\prime}(t) & =f(t, x(t))+\Delta(x), \quad t \in[0, T], \\
x(0) & =z .
\end{aligned}
$$

where the functional given by the formula $\Delta(x):=-T^{-1} \int_{0}^{T} f(s, x(s)) d s$ plays the role of a "perturbation term." The choice of such perturbation terms is, of course, not unique. Instead of problem (1.3), or, equivalently, of the integral functional equation

$$
x(t)=z+\int_{0}^{t} f(s, x(s)) d s-\frac{t}{T} \int_{0}^{T} f(s, x(s)) d s, \quad t \in[0, T],
$$

one can also consider the system

$$
x(t)=z+\int_{0}^{t} f(s, x(s)) d s-\omega(t) \int_{0}^{T} f(s, x(s)) d s, \quad t \in[0, T],
$$

where $\omega:[0, T] \rightarrow[0, T]$ is a continuous function with the properties $\omega(0)=0$ and $\omega(T)=1$, the arbitrariness of which in some cases [8] allows one to drop the smallness restriction of the Lipschitz constants involved.

A similar procedure also applies in other situtations. For example, in the case of the two-point boundary value problem

$$
A x(0)+B x(T)=d
$$

for equation (1.1), where $\{A, B\} \subset \mathcal{L}\left(\mathbb{R}^{n}\right), d \in \mathbb{R}^{n}$, det $B \neq 0$, the corresponding parametrized family of auxiliary differential equations can be chosen [23] in the form (1.3) with

$$
\Delta(x):=-\frac{1}{T} \int_{0}^{T} f(s, x(s, z)) d s+\frac{1}{T}\left(B^{-1} d-\left(B^{-1} A+\mathbb{1}_{n}\right) z\right),
$$

or, equivalently, as the equation

$$
\begin{aligned}
x(t)=z+\int_{0}^{t} f(s, x(s)) & d s-\frac{t}{T} \int_{0}^{T} f(s, x(s)) d s \\
+ & \frac{t}{T}\left(B^{-1} d-\left(B^{-1} A+\mathbb{1}_{n}\right) z\right), \quad t \in[0, T] .
\end{aligned}
$$

The solution of problem (1.3) in each case is sought for in an analytic form by the method of successive approximations similar to the Picard iterations. All the iterations $x_{m}(\cdot, z), m=1,2, \ldots$, depend upon the parameter $z$ and, for arbitrary its values, satisfy the given boundary conditions. For the periodic boundary value 
problem (1.1), (1.2), according to (1.4), the successive approximations have the form [19-22]

$$
x_{m}(t, z):=z+\int_{0}^{t} f\left(s, x_{m-1}(s, z)\right) d s-\frac{t}{T} \int_{0}^{T} f\left(s, x_{m-1}(s, z)\right) d s, \quad m \geq 1,
$$

where $x_{0}(t, z):=z, t \in[0, T]$, and have the properties

$$
x_{m}(0, z)=x_{m}(T, z)=z, \quad m=1,2, \ldots
$$

Sequence (1.8) is a particular case of the iteration process

$$
\begin{aligned}
x_{m}(t, z):=z+\int_{0}^{t}(f & \left.\left(s, x_{m-1}(s, z)\right)-\frac{1}{T} \int_{0}^{T} f\left(s, x_{m-1}(s, z)\right) d s\right) d s \\
& +\frac{t}{T}\left(B^{-1} d-\left(B^{-1} A+\mathbb{1}_{n}\right) z\right), \quad m=1,2, \ldots,
\end{aligned}
$$

used in [23] in the case of the two-point boundary value problem (1.1), (1.5). Here, $x_{0}(t, z):=z, t \in[0, T]$, and

$$
x_{m}(0, z)=z, \quad A x_{m}(0, z)+B x_{m}(T, z)=d
$$

for all $m=1,2, \ldots$.

The procedure of passing from the original differential system (1.1) to its "perturbed" counterpart (1.3) and the investigation of the latter by using successive approximations leads one to a certain system of algebraic or transcendental "determining equations," which give those numerical values $z=z^{*}$ of the parameter that correspond to the solutions of the given boundary value problem. For example, the determining system corresponding to the successive approximations above has the form

$$
\int_{0}^{T} f\left(s, x^{*}(s, z)\right) d s=B^{-1} d-\left(B^{-1} A+\mathbb{1}_{n}\right) z,
$$

for the two-point boundary value problem (1.1), (1.5) and

$$
\int_{0}^{T} f\left(s, x^{*}(s, z)\right) d s=0,
$$

in the case of the periodic boundary value problem $(1.1),(1.2)$, where $x^{*}(\cdot, z)$ is the limit function of sequences (1.8) and (1.9), respectively. The idea to introduce a parameter of the kind indicated resembles the Lyapunov-Schmidt approach (see, e.g., $[2,18])$, with the important difference that the resulting approximations automatically satisfy the given boundary conditions.

In some related works auxiliary equations are constructed in a different way. For instance, after the change of variable

$$
x(t)=z+\int_{0}^{t} y(s, z) d s-\frac{t}{T} \int_{0}^{T} y(s, z) d s,
$$


instead of the integral functional equation (1.4), one arrives at the equation

$$
y(t, z)=f\left(t, z+\int_{0}^{t} y(s, z) d s-\frac{t}{T} \int_{0}^{T} y(s, z) d s\right), \quad t \in[0, T],
$$

which should be considered together with the determining equation

$$
\int_{0}^{T} y(s, z) d s=0 .
$$

Analogously, the substitution

$$
x(t, z)=z+\int_{0}^{t} y(s, z) d s-\frac{t}{T} \int_{0}^{T} y(s, z) d s+\frac{t}{T}\left(B^{-1} d-\left(B^{-1} A+I\right) z\right)
$$

brings equation (1.7) to the form

$$
\begin{aligned}
y(t, z)=f\left(t, z+\int_{0}^{t} y(s, z) d s-\frac{t}{T} \int_{0}^{T} y(s, z) d s\right. & \\
& \left.+\frac{t}{T}\left(B^{-1} d-\left(B^{-1} A+I\right) z\right)\right)
\end{aligned}
$$

with the determining equations

$$
\int_{0}^{T} y(s, z) d s=B^{-1} d-\left(B^{-1} A+I\right) z .
$$

Equations of type (1.14) was used in [1,4-6] in studies of the two-point boundary value problem (1.5) for the neutral type functional differential equation

$$
x^{\prime}(t)=f\left(t, x(\alpha(t)), x^{\prime}(\beta(t))\right), \quad t \in[0, T],
$$

where $\alpha, \beta:[0, T] \rightarrow[0, T]$ are given continuous functions, $\{A, B\} \subset \mathcal{L}\left(\mathbb{R}^{n}\right), \operatorname{det} B \neq$ $0, d \in \mathbb{R}^{n}$, and $f:[0, T] \times \mathbb{R}^{n} \times \mathbb{R}^{n} \rightarrow \mathbb{R}^{n}$ is continuous in the first argument and for all $u, v$ satisfies the Lipschitz condition

$$
\left|f\left(t, u_{1}, v_{1}\right)-f\left(t, u_{2}, v_{2}\right)\right| \leq K\left|u_{1}-u_{2}\right|+L\left|v_{1}-v_{2}\right|
$$

with certain non-negative constant matrices $\{K, L\} \subset \mathcal{L}\left(\mathbb{R}^{n}\right)$. The approach to problem (1.16), (1.5) developed in [1] is based upon finding a solution $y=y^{*}(\cdot, z)$ of the equation

$$
\begin{aligned}
y(t, z)=f\left(t, z+\frac{\alpha(t)}{T} B^{-1}[d-(A+B) z]+\frac{T-\alpha(t)}{T} \int_{0}^{\alpha(t)} y(s, z) d s\right. & \\
& \left.-\frac{\alpha(T)}{T} \int_{\alpha(t)}^{T} y(s, z) d s, y(\beta(t), z)\right), \quad t \in[0, T],
\end{aligned}
$$


as the uniform limit of successive approximations, after which the value $z=z^{*}$ of the vector parameter $z$ is determined from equations (1.15). Of course, certain assumptions are necessary to ensure the applicability of such a scheme. The successive approximations constructed in [1] for problem (1.16), (1.5) converge if

$$
r\left(2 \sup _{t \in[0, T]}\left(1-T^{-1} \alpha(t)\right) \alpha(t) K+L\right)<1 .
$$

Note that $\max _{t \in[0, T]}\left(1-T^{-1} \alpha(t)\right) \alpha(t) \leq \max _{t \in[0, T]}\left(1-T^{-1} t\right) t=\frac{T}{4}$ and, therefore, if the range of the function $\alpha$ in (1.16) contains values greater than or equal to $\frac{T}{2}$, then inequality (1.17) takes the form

$$
r\left(\frac{T}{2} K+L\right)<1
$$

The applicability of the approach based on a substitution of type (1.13) to the more general system of functional differential equations

$$
\begin{aligned}
x^{\prime}(t)=f\left(t, x\left(\alpha_{1}(t)\right),\right. & x\left(\alpha_{2}(t)\right), \ldots, x\left(\alpha_{p}(t)\right), \\
& \left.x^{\prime}\left(\beta_{1}(t)\right), x^{\prime}\left(\beta_{2}(t)\right), \ldots, x^{\prime}\left(\beta_{q}(t)\right)\right)
\end{aligned}
$$

with the boundary conditions (1.5) is guaranteed in [1] by rather restrictive conditions (see [1, Assumptions $H_{1}^{\prime}$ and $\left.H_{2}^{\prime}\right]$ ).

In the present paper, we suggest a refinement of certain estimates related to the analysis of convergence of successive approximations of type (1.9) associated with a two-point boundary value problem for a class of systems of linear functional differential equations involving argument deviations that possess certain special properties.

\section{NOTATION}

The following notation is used in the sequel.

(1) $C\left([0, T], \mathbb{R}^{n}\right)$ is the Banach space of the continuous functions $[0, T] \rightarrow \mathbb{R}^{n}$ with the standard uniform norm.

(2) $L_{1}\left([0, T], \mathbb{R}^{n}\right)$ is the usual Banach space of the vector functions $[0, T] \rightarrow \mathbb{R}^{n}$ with Lebesgue integrable components.

(3) $\mathcal{L}\left(\mathbb{R}^{n}\right)$ is the algebra of square matrices of dimension $n$ with real elements.

(4) $r(A)$ is the maximal in modulus eigenvalue of a matrix $A \in \mathcal{L}\left(\mathbb{R}^{n}\right)$.

(5) $\mathbb{1}_{n}$ is the unit matrix of dimension $n$.

\section{Problem Setting}

We consider the system of linear differential equations with argument deviations

$$
x^{\prime}(t)=P_{0}(t) x(t)+P_{1}(t) x(\beta(t))+f(t), \quad t \in[0, T],
$$

subjected to the inhomogeneous two-point boundary conditions of the form

$$
A x(0)+B x(T)=d .
$$


For the sake of simplicity, we restrict ourselves to the case where only one argument deviation is present. Here, $T \in(0,+\infty)$, the elements of the matrix-valued functions $P_{i}:[0, T] \rightarrow \mathcal{L}\left(\mathbb{R}^{n}\right), i=0,1$, are Lebesgue integrable, $f \in L_{1}\left([0, T], \mathbb{R}^{n}\right),\{A, B\} \subset$ $\mathcal{L}\left(\mathbb{R}^{n}\right), d \in \mathbb{R}^{n}$, and $\beta:[0, T] \rightarrow[0, T]$ is a Lebesgue measurable function. In the continuously differentiable case, problem (3.1), (3.2) is a particular case of the nonlinear boundary value problem (1.19), (1.5) considered in [1] for

$$
\alpha_{1}(t):=t, \quad \alpha_{2}(t):=\beta(t), \quad t \in[0, T] .
$$

The aim of this paper is to suggest a scheme for investigation of the two-point problem (3.1), (3.2) based upon equations of type (1.7), (1.11), which are constructed without application of substitution (1.13) and equation (1.14). In particular, it will be shown that, for certain classes of argument deviations $\beta$ in the differential system (3.1), the corresponding iteration process for problem (3.1), (3.2) is convergent provided that

$$
r\left(K_{1}+K_{2}\right)<\frac{10}{3 T}
$$

where $K_{i}:=\operatorname{ess~sup}_{t \in[0, T]}\left|P_{i}(t)\right|, i=0,1$.

Note that, in the case of problem (3.1), (3.2), the assumptions of [1], in particular, imply that the inequalities

$$
r\left(d_{1} K_{1}+d_{2} K_{2}\right)<1
$$

and

$$
r\left(T\left|(A+B)^{-1} B\right| C^{-1} \sum_{i=1}^{2} K_{i} \sup _{t \in[0, T]}\left|\mathbb{1}_{n}-\frac{\alpha_{i}(t)}{T} B^{-1}(A+B)\right|\right)<1
$$

are satisfied, where $C:=\mathbb{1}_{n}-\sum_{i=1}^{2} d_{i} K_{i}$, the functions $\alpha_{i}, i=1,2$, are given by (3.3), and

$$
d_{i}:=\frac{2}{T} \sup _{t \in[0, T]}\left(T-\alpha_{i}(t)\right) \alpha_{i}(t), \quad i=1,2 .
$$

These conditions are usually stronger than condition (3.4). To notice this, it sufficient to compare (3.4) with (3.5) and notice, e. g., that, in the case indicated, $d_{1}=\frac{T}{2}$ and, therefore, condition (3.5) has the form

$$
r\left(K_{1}+\frac{4}{T^{2}} \sup _{t \in[0, T]}(T-\beta(t)) \beta(t) K_{2}\right)<\frac{2}{T} .
$$




\section{FORMULAE FOR THE ITERATION PROCESS}

With the boundary value problem (3.1), (3.2), we associate the sequence of continuous functions defined by the recurrence relation

$$
\begin{array}{r}
x_{m+1}(t, z):=z+\int_{0}^{t}\left[P_{0}(s) x_{m}(s, z)+P_{1}(s) x_{m}(\beta(s), z)+f(s)\right] d s- \\
-\frac{t}{T} \int_{0}^{T}\left[P_{0}(s) x_{m}(s, z)+P_{1}(s) x_{m}(\beta(s), z)+f(s)\right] d s \\
+\frac{t}{T}\left(B^{-1} d-\left(B^{-1} A+\mathbb{1}_{n}\right) z\right),
\end{array}
$$

where $m=0,1,2, \ldots$ and $x_{0}(\cdot, z)=z$ for all $z \in \mathbb{R}^{n}$.

Proposition 1. All the members of sequence (4.1), starting with the first one, are absolutely continuous functions satisfying the boundary conditions (3.2) for an arbitrary $z \in \mathbb{R}^{n}$ :

$$
A x_{m}(0, z)+B x_{m}(T, z)=d, \quad m=1,2, \ldots, z \in \mathbb{R}^{n},
$$

an the initial condition

$$
x_{m}(0, z)=z, \quad m=1,2, \ldots, z \in \mathbb{R}^{n} .
$$

Proof. The statement indicated is an immediate consequence of formula (4.1) and the form of function $x_{0}$.

Let $G \in \mathcal{L}\left(\mathbb{R}^{n}\right)$ and $g:[0, T] \times \mathbb{R}^{n} \rightarrow[0,+\infty)$.

Definition 1. We say that the successive approximation method (4.1) for problem (3.1), (3.2) is applicable with an estimate of the type $(G, g)$ if

(1) $r(G)<1$;

(2) $\sup _{t \in[0, T]} g(t, z)<+\infty$ for all $z \in \mathbb{R}^{n}$;

(3) For any $z \in \mathbb{R}^{n}$ there exists a continuous function $x^{*}(\cdot, z):[0, T] \rightarrow \mathbb{R}^{n}$ such that the pointwise and componentwise estimates

$$
\left|x^{*}(t, z)-x_{m}(t, z)\right| \leq G^{m}\left(\mathbb{1}_{n}-G\right)^{-1} g(t, z), \quad t \in[0, T], m=1,2, \ldots,
$$

are true.

Proposition 2. Let for certain $G \in \mathcal{L}\left(\mathbb{R}^{n}\right)$ and $g:[0, T] \times \mathbb{R}^{n} \rightarrow[0,+\infty)$ the successive approximation method (4.1) be applicable to problem (3.1), (3.2) with an estimate of the type $(G, g)$. Then:

(1) For any fixed $z \in \mathbb{R}^{n}$, the function $x^{*}:[0, T] \times \mathbb{R}^{n} \rightarrow \mathbb{R}^{n}$ appearing in (4.4) coincides with the uniform limit of sequence (4.1):

$$
\lim _{m \rightarrow \infty} \max _{t \in[0, T]}\left|x^{*}(t, z)-x_{m}(t, z)\right|=0 .
$$


(2) The limit function of sequence (4.1),

$$
x^{*}(t, z)=\lim _{m \rightarrow \infty} x_{m}(t, z),
$$

for an arbitrary $z \in \mathbb{R}^{n}$ satisfies the initial condition

$$
x^{*}(0, z)=z
$$

and the boundary conditions (3.2):

$$
A x^{*}(0, z)+B x^{*}(T, z)=d, \quad z \in \mathbb{R}^{n} .
$$

(3) Function (4.5) is an absolutely continuous solution of the integro-functional equation

$$
\begin{aligned}
x(t)=z+\int_{0}^{t}\left[P_{0}(s) x(s)+\right. & \left.P_{1}(s) x(\beta(s))+f(s)\right] d s \\
-\frac{t}{T} \int_{0}^{T}\left[P_{0}(s) x(s)+P_{1}(s) x(\beta(s))+f(s)\right] d s & \\
& +\frac{t}{T}\left[B^{-1} d-\left(B^{-1} A+\mathbb{1}_{n}\right) z\right], \quad t \in[0, T],
\end{aligned}
$$

or, which is equivalent, of the Cauchy problem (4.6) for the functional differential equation

$$
\begin{aligned}
& x^{\prime}(t)=P_{0}(t) x(t)+P_{1}(t) x(\beta(t))+f(t)+\Delta(x ; z), \quad t \in[0, T], \\
& \text { where } \\
& \Delta(x ; z):=\frac{1}{T}\left[B^{-1} d-\left(B^{-1} A+\mathbb{1}_{n}\right) z\right] \\
& -\frac{1}{T} \int_{0}^{T}\left[P_{0}(s) x(s)+P_{1}(s) x(\beta(s))+f(s)\right] d s, \quad z \in \mathbb{R}^{n} .
\end{aligned}
$$

Proof. This assertion is easily obtained by taking Definition 1 and Proposition 1 into account.

Let us formulate a general statement which establishes a relation between the limit function $x^{*}$ of the sequence (4.1) to the solutions of the two-point problem (3.1), (3.2). For this purpose, consider the Cauchy problem

$$
\begin{gathered}
x^{\prime}(t)=P_{0}(t) x(t)+P_{1}(t) x(\beta(t))+f(t)+\mu, \quad t \in[0, T], \\
x(0)=z,
\end{gathered}
$$

where $\mu \in \mathbb{R}^{n}$ and $z \in \mathbb{R}^{n}$ are certain parameters.

Proposition 3. Let us assume that the successive approximation method (4.1) for problem (3.1), (3.2) is applicable with an estimate of the type $(G, g)$ for certain $G \in$ 
$\mathcal{L}\left(\mathbb{R}^{n}\right)$ and $g:[0, T] \times \mathbb{R}^{n} \rightarrow[0,+\infty)$. Then the solution $x(\cdot)$ of the initial value problem (4.10), (4.11) satisfies the two-point boundary condition (3.2) if, and only if

$$
\begin{aligned}
\mu=\frac{1}{T}\left[B^{-1} d\right. & \left.-\left(B^{-1} A+\mathbb{1}_{n}\right) z\right] \\
& -\frac{1}{T} \int_{0}^{T}\left[P_{0}(s) x^{*}(s, z)+P_{1}(s) x^{*}(\beta(s), z)+f(s)\right] d s,
\end{aligned}
$$

where $x^{*}(\cdot, z):[0, T] \rightarrow \mathbb{R}^{n}$ is the function given by (4.5).

Proof. By virtue of Proposition 2, for an arbitrary $z \in \mathbb{R}^{n}$, the function $x^{*}(\cdot, z)$ defined by formula (4.5) satisfies the integro-functional equation (4.7). Differentiating (4.7), we find that $x=x^{*}(\cdot, z)$ is a solution of the Cauchy problem (4.10), (4.11) with $\mu$ given by equality (4.12).

Proposition 3 implies immediately the following

Proposition 4. Let the successive approximation method (4.1) be applicable to problem (3.1), (3.2) with an estimate of the type $(G, g)$ for certain $G \in \mathcal{L}\left(\mathbb{R}^{n}\right)$ and $g:[0, T] \times \mathbb{R}^{n} \rightarrow[0,+\infty)$. Then the limit function $x^{*}(\cdot, z)$ of the recurrence sequence (4.1) is a solution of the boundary value problem (3.1), (3.2) if, and only if the value of the vector parameter $z \in \mathbb{R}^{n}$ in (4.1) satisfies the system of equations

$$
\begin{aligned}
\left(B^{-1} A+\mathbb{1}_{n}\right) z= & B^{-1} d \\
& -\int_{0}^{T}\left[P_{0}(s) x^{*}(s, z)+P_{1}(s) x^{*}(\beta(s), z)+f(s)\right] d s .
\end{aligned}
$$

Proof. It suffices to apply Proposition 3 and notice that equation (4.8) coincides with equation (3.1) if and only if relation (4.13) holds.

Equations of type (4.13) in Proposition 4 are often called determining equations (see, e. g., [3]), because it is from them one has to determine the actual values of the parameters involved in the iteration process.

Remark 1. In practice, it is convenient to fix some $m$ and consider the "approximate determining equation"

$$
B^{-1} d-\left(B^{-1} A+\mathbb{1}_{n}\right) z=\int_{0}^{T}\left[A(s) x_{m}(s, z)+B(s) x_{m}(\beta(s), z)+f(s)\right] d s .
$$

If equation (4.14) has an isolated solution $z=z_{m}$ in a certain open domain $D \subset$ $\mathbb{R}^{n}$, under additional assumptions one can show that the corresponding exact system of determining equations (4.13) is also solvable and, therefore, by virtue of Proposition 4, the boundary value problem (3.1), (3.2) has a solution (see, e. g., Theorem 3.1 in [10] or Theorem 7.1 in [22]). In this case, the function

$$
[0, T] \ni t \longmapsto X_{m}(t):=x_{m}\left(t, z_{m}\right)
$$


can be regarded as the mth approximation to a solution of problem (3.1), (3.2).

\section{Auxiliary STATEMENTS}

Let us define the sequence of functions $\left\{\alpha_{m}\right\}_{m=0}^{\infty} \subset C([0, T], \mathbb{R})$ by the recurrence relation

$$
\alpha_{m}(t)=\left(1-\frac{t}{T}\right) \int_{0}^{t} \alpha_{m-1}(s) d s+\frac{t}{T} \int_{t}^{T} \alpha_{m-1}(s) d s, \quad t \in[0, T],
$$

for $m=1,2, \ldots$, where $\alpha_{0}(t):=1, t \in[0, T]$. It is obvious that, in particular,

$$
\alpha_{1}(t)=2 t\left(1-\frac{t}{T}\right), \quad t \in[0, T],
$$

and

$$
\max _{t \in[0, T]} \alpha_{1}(t)=\frac{T}{2} .
$$

Lemma 1. Each of functions (5.1) is non-negative, takes zero values at the points 0 and $T$, and possesses the property

$$
\alpha_{m}\left(\frac{T}{2}-t\right)=\alpha_{m}\left(\frac{T}{2}+t\right), \quad m \geq 1, t \in[0, T / 2] .
$$

Moreover,

$$
\alpha_{m}^{\prime}(t) \operatorname{sign}\left(t-\frac{T}{2}\right) \leq 0, \quad t \in[0, T], m=1,2, \ldots, n .
$$

Proof. The first assertion is an immediate consequence of [7, Lemma 1]. Let us verify that $\alpha_{m}$ is non-decreasing on $[0, T / 2]$ for any $m \geq 1$. Indeed, it follows from (5.1) and the non-negativity of $\alpha_{m}$ that

$$
\begin{aligned}
\alpha_{m}^{\prime}(t) & =\frac{1}{T}\left(-\int_{0}^{t} \alpha_{m-1}(s) d s+\int_{t}^{T} \alpha_{m-1}(s) d s\right)+\left(1-\frac{2 t}{T}\right) \alpha_{m-1}(t) \\
& =\frac{1}{T}\left(\int_{0}^{T} \alpha_{m-1}(s) d s-2 \int_{0}^{t} \alpha_{m-1}(s) d s\right)+\left(1-\frac{2 t}{T}\right) \alpha_{m-1}(t) . \\
& \geq \frac{1}{T}\left(\int_{0}^{T} \alpha_{m-1}(s) d s-2 \int_{0}^{\frac{T}{2}} \alpha_{m-1}(s) d s\right)+\left(1-\frac{2 t}{T}\right) \alpha_{m-1}(t)
\end{aligned}
$$

for any $t \in[0, T / 2]$ and $m \geq 1$. On the other hand, relation (5.4) of Lemma 1 implies that

$$
\int_{0}^{\frac{T}{2}} \alpha_{m-1}(s) d s=\int_{\frac{T}{2}}^{T} \alpha_{m-1}(s) d s, \quad m=1,2, \ldots,
$$




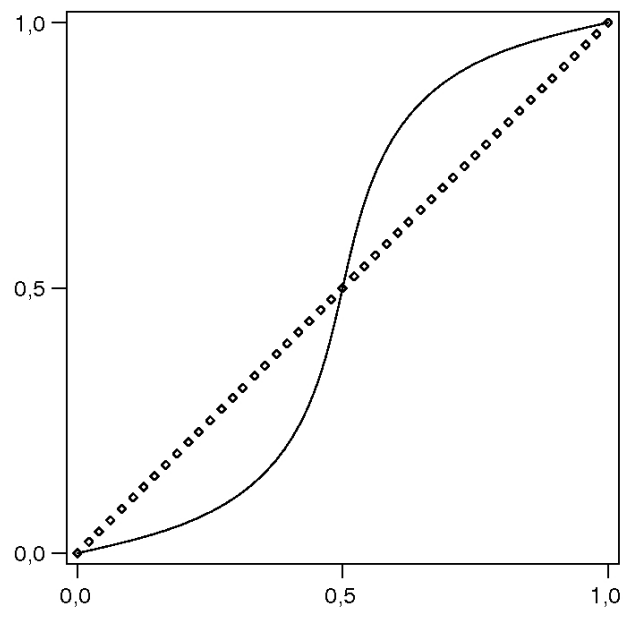

FIGURE 1. An example of an argument transformation $\beta$ satisfying condition (5.8) for $T=1$

and, therefore, (5.6) yields the estimate

$$
\alpha_{m}^{\prime}(t) \geq \frac{1}{T}\left(-\int_{0}^{\frac{T}{2}} \alpha_{m-1}(s) d s+\int_{\frac{T}{2}}^{T} \alpha_{m-1}(s) d s\right)+\left(1-\frac{2 t}{T}\right) \alpha_{m-1}(t) \geq 0
$$

for $t \in[0, T / 2]$ and $m \geq 1$. The required relation (5.5) now follows from the symmetry property (5.2).

Lemma 2. For an arbitrary essentially bounded function $u:[0, T] \rightarrow \mathbb{R}$, the estimate

$$
\left|\int_{0}^{t}\left(u(\tau)-\frac{1}{T} \int_{0}^{T} u(s) d s\right) d \tau\right| \leq \frac{1}{2} \alpha_{1}(t)(\underset{s \in[0, T]}{\operatorname{ess} \sup u(s)-\underset{s \in[0, T]}{\operatorname{essinf}} u(s)})
$$

is true for all $t \in[0, T]$, where $\alpha_{1}$ is the function defined by equality (5.2).

Inequality (5.7) is established similarly to the Lemma from [9], and the explicit proof is therefore omitted.

Lemma 3. Let $\beta:[0, T] \rightarrow[0, T]$ be a measurable function satisfying the condition

$$
\underset{t \in[0, T]}{\operatorname{essinf}}(\beta(t)-t) \operatorname{sign}\left(t-\frac{T}{2}\right) \geq 0 .
$$

Then the members of the function sequence (5.1) satisfy the pointwise estimates

$$
\alpha_{m}(\beta(t)) \leq \alpha_{m}(t), \quad m=1,2, \ldots, t \in[0, T] .
$$


Proof. We shall use properties of functions (5.1). Indeed, it follows from Lemma 1 that (5.5) holds for any $t \in[0, T]$ and $m \geq 1$, i. e., each $\alpha_{m}, m=1,2, \ldots$, is nondecreasing on the interval $[0, T / 2]$ and non-increasing on $[T / 2, T]$. Combining (5.5) and (5.8), we easily obtain the required estimate (5.9).

Note that if $\beta:[0, T] \rightarrow[0, T]$ is a continuous function satisfying condition (5.8) then necessarily $\beta(0)=0, \beta\left(\frac{T}{2}\right)=\frac{T}{2}$, and $\beta(T)=T$.

Lemma 4. If a measurable function $\beta:[0, T] \rightarrow[0, T]$ satisfies the condition

$$
k_{T}(\beta):=\operatorname{esssup}_{t \in(0, T)} \frac{\beta(t)(T-\beta(t))}{t(T-t)}<+\infty
$$

then

$$
\alpha_{1}(\beta(t)) \leq k_{T}(\beta) \alpha_{1}(t), \quad t \in[0, T] .
$$

Proof. The assertion of Lemma 4 follows immediately from (5.10) and properties of function (5.2).

It should be noted that (5.10) does not imply the condition $k_{T}(\lambda \beta)<+\infty$, where $\lambda \in \mathbb{R}$.

Example 1. The function $\beta(t)=t^{2}, t \in[0,1]$, satisfies condition (5.10) on the interval $[0,1]$. However, the condition mentioned is not satisfied for none of the functions $\beta(t)=\frac{1}{2} t^{2}, \beta(t)=\frac{t}{2}$, and $\beta(t)=\sin t, t \in[0,1]$. This fact is a consequence of the elementary relations

$$
\sup _{t \in(0, T)} \frac{t^{2}\left(1-t^{2}\right)}{t(1-t)}=2, \quad \quad \lim _{t \rightarrow 1^{-}} \frac{\frac{t^{2}}{2}\left(1-\frac{t^{2}}{2}\right)}{t(1-t)}=+\infty
$$

and

$$
\lim _{t \rightarrow 1^{-}} \frac{\frac{t}{2}\left(1-\frac{t}{2}\right)}{t(1-t)}=+\infty, \quad \quad \lim _{t \rightarrow 1^{-}} \frac{\sin t(1-\sin t)}{1-t}=+\infty .
$$

6. CONVERGENCE OF SUCCESSIVE APPROXIMATIONS FOR THE CASE OF A GENERAL TYPE OF ARGUMENT DEVIATION

Theorem 1. Let $P_{i}:[0, T] \rightarrow \mathcal{L}\left(\mathbb{R}^{n}\right), i=0,1$, be matrix-valued functions with essentially bounded elements, the function $\beta:[0, T] \rightarrow[0, T]$ be measurable, and the matrix $B$ be non-singular. Let, moreover,

$$
r\left(\widetilde{P}_{0}+\widetilde{P}_{1}\right)<\frac{2}{T}
$$

where

$$
\widetilde{P}_{i}:=\underset{t \in[0, T]}{\operatorname{ess} \sup }\left|P_{i}(t)\right|, \quad i=0,1
$$


Then the successive approximation method (4.1) is applicable to problem (3.1), (3.2) with an estimate of the type $\left(T\left(\widetilde{P}_{0}+\widetilde{P}_{1}\right) / 2, \gamma\right)$, where

$$
\gamma(z):=\frac{T}{2} \delta(z)+\left|B^{-1} d-\left(B^{-1} A+\mathbb{1}_{n}\right) z\right|
$$

and

$$
\begin{aligned}
\delta(z):=\frac{1}{2}\left(\begin{array}{l}
\operatorname{ess} \sup \left(P_{0}(t) z+P_{1}(t) z\right. \\
t \in[0, T]
\end{array}\right. & f(t)) \\
& \left.\quad-\operatorname{essinf}_{t \in[0, T]}\left(P_{0}(t) z+P_{1}(t) z+f(t)\right)\right)
\end{aligned}
$$

for all $z \in \mathbb{R}^{n}$.

The symbols essinf, ess sup, $|\cdot|$, and $\leq$, in (6.2) and similar relations for vector and matrix-valued functions are understood in the componentwise sense (e.g., if $P=\left(p_{i j}\right)_{i, j=1}^{n}:[0, T] \rightarrow \mathscr{L}\left(\mathbb{R}^{n}\right)$, then ess $\sup _{t \in[0, T]}|P(t)|$ is the matrix with the components ess $\sup _{t \in[0, T]}\left|p_{i j}(t)\right|, i, j=1,2, \ldots, n$, and so on).

Proof. Let us show that, under the conditions assumed, sequence (4.1) is a Cauchy sequence in $C\left([0, T], \mathbb{R}^{n}\right)$. Due to estimate (5.7) of Lemma 2, it follows from (4.1) that for $m=0$ and an arbitrary fixed $z \in \mathbb{R}^{n}$

$$
\begin{array}{r}
\left|x_{1}(t, z)-z\right|=\mid \int_{0}^{t}\left(Q(s) z+f(s)-\frac{1}{T} \int_{0}^{T}(Q(\tau) z+f(\tau)) d \tau\right) d s \\
+\frac{t}{T}\left[B^{-1} d-\left(B^{-1} A+\mathbb{1}_{n}\right) z\right] \mid \leq \alpha_{1}(t) \delta(z)+\delta_{1}(z),
\end{array}
$$

where $Q:=P_{0}+P_{1}, \alpha_{1}$ is the function given by (5.2), and

$$
\delta_{1}(z):=\left|B^{-1} d-\left(B^{-1} A+\mathbb{1}_{n}\right) z\right|, \quad z \in \mathbb{R}^{n} .
$$

According to formulae (4.1), for all $t \in[0, T], z \in \mathbb{R}^{n}$ and $m=1,2, \ldots$ we have

$$
\begin{aligned}
r_{m+1}(t, z)= & \int_{0}^{t}\left[P_{0}(s) r_{m}(s, z)+P_{1}(s) r_{m}(\beta(s), z)\right] d s- \\
& -\frac{t}{T} \int_{0}^{T}\left[P_{0}(s) r_{m}(s, z)+P_{1}(s) r_{m}(\beta(s), z)\right] d s= \\
= & \left(1-\frac{t}{T}\right) \int_{0}^{t}\left[P_{0}(s) r_{m}(s, z)+P_{1}(s) r_{m}(\beta(s), z)\right] d s- \\
& -\frac{t}{T} \int_{t}^{T}\left[P_{0}(s) r_{m}(s, z)+P_{1}(s) r_{m}(\beta(s), z)\right] d s,
\end{aligned}
$$


where

$$
r_{m}(t, z):=x_{m}(t, z)-x_{m-1}(t, z), \quad t \in[0, T], m=1,2, \ldots,
$$

Equalities (6.7) imply that, for all $m=1,2, \ldots, z \in \mathbb{R}^{n}$ and all $t \in[0, T]$,

$$
\begin{aligned}
\left|r_{m+1}(t, z)\right| & \leq \widetilde{P}_{0}\left[\left(1-\frac{t}{T}\right) \int_{0}^{t}\left|r_{m}(s, z)\right| d s+\frac{t}{T} \int_{t}^{T}\left|r_{m}(s, z)\right| d s\right]+ \\
+ & \widetilde{P}_{1}\left[\left(1-\frac{t}{T}\right) \int_{0}^{t}\left|r_{m}(\beta(s), z)\right| d s+\frac{t}{T} \int_{t}^{T}\left|r_{m}(\beta(s), z)\right| d s\right],
\end{aligned}
$$

where $\widetilde{P}_{0}$ and $\widetilde{P}_{1}$ are the non-negative matrices defined by formula (6.2). Relation (6.5) yields

$$
\left|r_{1}(t, z)\right|=\left|x_{1}(t, z)-z\right| \leq \alpha_{1}(t) \delta(z)+\delta_{1}(z), \quad t \in[0, T],
$$

where $\delta_{1}$ is given by (6.6). In view of property (5.3) of the function $\alpha_{1}$, estimate (6.10) gives

$$
\left|r_{1}(t, z)\right| \leq \frac{T}{2} \delta(z)+\delta_{1}(z)=\gamma(z), \quad t \in[0, T] .
$$

Let us now estimate $r_{2}(t, z)$ using (6.9) and (6.11):

$$
\begin{aligned}
\left|r_{2}(t, z)\right| \leq & \widetilde{P}_{0}\left(\left(1-\frac{t}{T}\right) \int_{0}^{t}\left|r_{1}(s, z)\right| d s+\frac{t}{T} \int_{t}^{T}\left|r_{1}(s, z)\right| d s\right) \\
+ & \widetilde{P}_{1}\left(\left(1-\frac{t}{T}\right) \int_{0}^{t}\left|r_{1}(\beta(s), z)\right| d s+\frac{t}{T} \int_{t}^{T}\left|r_{1}(\beta(s), z)\right| d s\right) \\
& \leq \widetilde{P}_{0}\left(\left(1-\frac{t}{T}\right) \int_{0}^{t} \gamma(z) d s+\frac{t}{T} \int_{t}^{T} \gamma(z) d s\right) \\
+ & \widetilde{P}_{1}\left(\left(1-\frac{t}{T}\right) \int_{0}^{t} \gamma(z) d s+\frac{t}{T} \int_{t}^{T} \gamma(z) d s\right), \quad t \in[0, T] .
\end{aligned}
$$

Taking the definitions (6.3) and (6.4) into account and using equality (5.3), from (6.12) we get

$$
\left|r_{2}(t, z)\right| \leq\left(\widetilde{P}_{0}+\widetilde{P}_{1}\right) \gamma(z) \alpha_{1}(t) \leq \frac{T}{2}\left(\widetilde{P}_{0}+\widetilde{P}_{1}\right) \gamma(z), \quad t \in[0, T] .
$$

Arguing by induction, we then obtain that, for all $t \in[0, T]$ and $m=1,2, \ldots$, the estimates

$$
\left|r_{m}(t, z)\right| \leq\left(\widetilde{P}_{0}+\widetilde{P}_{1}\right)^{m-1}\left(\frac{T}{2}\right)^{m-1} \gamma(z)=G^{m-1} \gamma(z),
$$


where

$$
G:=\frac{T}{2}\left(\widetilde{P}_{0}+\widetilde{P}_{1}\right)
$$

are true. Estimate (6.13), according to definition (6.8), yields

$$
\left|x_{m+j}(t, z)-x_{m}(t, z)\right| \leq \sum_{i=1}^{j}\left|r_{m+i}(t, z)\right| \leq G^{m} \sum_{i=0}^{j-1} G^{i} \gamma(z)
$$

for $t \in[0, T], m=1,2, \ldots$, whence, by virtue of assumption (6.1) and notation (6.14), it follows that

$$
\left|x_{m+j}(t, z)-x_{m}(t, z)\right| \leq G^{m} \sum_{i=0}^{\infty} G^{i} \gamma(z)=G^{m}\left(\mathbb{1}_{n}-G\right)^{-1} \gamma(z)
$$

for all $t \in[0, T]$ and $m=1,2, \ldots$. Since, due to (6.1), $G^{m} \rightarrow 0$ for $m \rightarrow \infty$, it is clear from (6.15) that (4.1) is a Cauchy sequence in $C\left([0, T], \mathbb{R}^{n}\right)$ and, therefore,

$$
\left|x^{*}(t, z)-x_{m}(t, z)\right| \leq G^{m}\left(\mathbb{1}_{n}-G\right)^{-1} \gamma(z)
$$

for all $t \in[0, T], z \in \mathbb{R}^{n}$ and $m=1,2, \ldots$. According to Definition 1, this leads us to the assertion of Theorem 1 .

Remark 2. In the case where the function $\beta$ is continuous and the set of its values contains numbers greater than or equal to $\frac{T}{2}$, condition (6.1), which guarantees the applicability of the successive approximation method (4.1), coincides with condition (1.7) from [1] .

\section{CONVERGENCE OF SUCCESSIVE APPROXIMATIONS FOR SPECIAL KINDS OF ARGUMENT DEVIATIONS}

In the cases where the function $\beta:[0, T] \rightarrow[0, T]$ satisfies one of conditions (5.8) and (5.10), the assumption (6.1) of Theorem 1 can be weakened.

Theorem 2. If the function $\beta:[0, T] \rightarrow[0, T]$ possesses property (5.8) and, moreover, the inequality

$$
r\left(\widetilde{P}_{0}+\widetilde{P}_{1}\right)<\frac{10}{3 T},
$$

is satisfied, then the successive approximation method (4.1) is applicable to problem (3.1), (3.2) with an estimate of the type $\left(3 T\left(\widetilde{P}_{0}+\widetilde{P}_{1}\right) / 10, g\right)$, where

$$
g(t, z):=\frac{100}{27 T} \alpha_{1}(t) \gamma(z), \quad t \in[0, T], z \in \mathbb{R}^{n},
$$

and the function $\gamma$ is defined by formula (6.3). 
Proof. It follows from Lemma 3 that condition (5.8) ensures the validity of estimate (5.9). Therefore,

$$
\alpha_{1}(\beta(t)) \leq \alpha_{1}(t), \quad t \in[0, T],
$$

where $\alpha_{1}$ is the function (5.2). Let us estimate the value $r_{2}(t, z)$. Using (6.9), (6.11), and (7.1), we have

$$
\begin{aligned}
\left|r_{2}(t, z)\right| & \leq \widetilde{P}_{0}\left(\left(1-\frac{t}{T}\right) \int_{0}^{t}\left(\frac{T}{2} \delta(z)+\delta_{1}(z)\right) d s+\frac{t}{T} \int_{t}^{T}\left(\frac{T}{2} \delta(z)+\delta_{1}(z)\right) d s\right) \\
& +\widetilde{P}_{1}\left(\left(1-\frac{t}{T}\right) \int_{0}^{t}\left(\frac{T}{2} \delta(z)+\delta_{1}(z)\right) d s+\frac{t}{T} \int_{t}^{T}\left(\frac{T}{2} \delta(z)+\delta_{1}(z)\right) d s\right) \\
& \leq\left(\widetilde{P}_{0}+\widetilde{P}_{1}\right) \gamma(z) \alpha_{1}(t)
\end{aligned}
$$

for all $t \in[0, T]$, where $\delta_{1}$ and $\gamma$ are defined by relations (6.6) and (6.3). From estimate (7.2), due to (7.1), we obtain

$$
\left|r_{2}(\beta(t), z)\right| \leq\left(\widetilde{P}_{0}+\widetilde{P}_{1}\right) \gamma(z) \alpha_{1}(\beta(t)) \leq\left(\widetilde{P}_{0}+\widetilde{P}_{1}\right) \gamma(z) \alpha_{1}(t), \quad t \in[0, T] .
$$

Arguing by induction, we arrive at the pointwise and coordinatewise estimates

$$
\left|r_{m+1}(t, z)\right| \leq\left(\widetilde{P}_{0}+\widetilde{P}_{1}\right)^{m} \gamma(z) \alpha_{m}(t), \quad t \in[0, T], m=1,2, \ldots,
$$

and

$$
\left|r_{m+1}(\beta(t), z)\right| \leq\left(\widetilde{P}_{0}+\widetilde{P}_{1}\right)^{m} \gamma(z) \alpha_{m}(t), \quad t \in[0, T], m=1,2, \ldots,
$$

where $\alpha_{m}, m=1,2, \ldots$, are the functions of sequence (5.1). By virtue of Lemma 2.4 from [10], the estimates

$$
\alpha_{m+1}(t) \leq \frac{3 T}{10} \alpha_{m}(t), m \geq 2, \quad \alpha_{m+1}(t) \leq\left(\frac{3 T}{10}\right)^{m} \bar{\alpha}_{1}(t), m \geq 0,
$$

are true for all $t \in[0, T]$ and $m \geq 1$, where

$$
\bar{\alpha}_{1}(t):=\frac{10}{9} \alpha_{1}(t), \quad t \in[0, T] .
$$

Taking (7.5) into account and using (7.3) and (7.4), arrive at the inequalities

$$
\left|r_{m+1}(t, z)\right| \leq\left(\widetilde{P}_{0}+\widetilde{P}_{1}\right)\left(\frac{3 T}{10}\left(\widetilde{P}_{0}+\widetilde{P}_{1}\right)\right)^{m-1} \gamma(z) \bar{\alpha}_{1}(t), \quad t \in[0, T], m \geq 1,
$$

and

$\left|r_{m+1}(\beta(t), z)\right| \leq\left(\widetilde{P}_{0}+\widetilde{P}_{1}\right)\left(\frac{3 T}{10}\left(\widetilde{P}_{0}+\widetilde{P}_{1}\right)\right)^{m-1} \gamma(z) \bar{\alpha}_{1}(t), \quad t \in[0, T], m \geq 1$.

The rest of the argument is very similar to that of the proof of Theorem 1 and, therefore, is omitted. 
The next statement deals with the case where the argument deviation $\beta$ in equation (3.1) satisfies condition (5.10).

Theorem 3. Let us assume that the function $\beta:[0, T] \rightarrow[0, T]$ possesses property (5.10) and, moreover, the inequality

$$
r\left(\widetilde{P}_{0}+\widetilde{P}_{1}\right)<\frac{3}{T \max \left\{1, k_{T}(\beta)\right\}},
$$

holds, where $k_{T}(\beta)$ is the positive constant appearing in relation (5.10).

Then the successive approximation method (4.1) is applicable to problem (3.1), (3.2) with an estimate of the type $\left(T \max \left\{1, k_{T}(\beta)\right\}\left(\widetilde{P}_{0}+\widetilde{P}_{1}\right) / 3, g\right)$, where

$$
g(t, z):=\frac{3 \alpha_{1}(t)}{T} \gamma(z), \quad t \in[0, T], z \in \mathbb{R}^{n},
$$

and $\gamma$ is given by formula (6.3).

Proof. Let us put $\eta:=\max \left\{1, k_{T}(\beta)\right\}$. According to (6.12), we have

$$
\left|r_{2}(t, z)\right| \leq\left(\widetilde{P}_{0}+\widetilde{P}_{1}\right) \gamma(z) \alpha_{1}(t) \leq \eta\left(\widetilde{P}_{0}+\widetilde{P}_{1}\right) \gamma(z) \alpha_{1}(t), \quad t \in[0, T]
$$

and, hence, in view of (5.11),

$$
\begin{array}{r}
\left|r_{2}(\beta(t), z)\right| \leq\left(\widetilde{P}_{0}+\widetilde{P}_{1}\right) \gamma(z) \alpha_{1}(\beta(t)) \leq k_{T}(\beta)\left(\widetilde{P}_{0}+\widetilde{P}_{1}\right) \gamma(z) \alpha_{1}(t) \\
\leq \eta\left(\widetilde{P}_{0}+\widetilde{P}_{1}\right) \gamma(z) \alpha_{1}(t), \quad t \in[0, T] .
\end{array}
$$

Using (6.9), (7.8), (7.9), (5.1), and (7.5) and carrying out calculations, we obtain

$$
\begin{aligned}
\left|r_{3}(t, z)\right| \leq & \eta \widetilde{P}_{0}\left(\widetilde{P}_{0}+\widetilde{P}_{1}\right) \gamma(z) \alpha_{2}(t)+\eta \widetilde{P}_{1}\left(\widetilde{P}_{0}+\widetilde{P}_{1}\right) \gamma(z) \alpha_{2}(t) \\
& \leq \eta\left(\widetilde{P}_{0}+\widetilde{P}_{1}\right)^{2} \gamma(z) \alpha_{2}(t) \leq \frac{T \eta}{3}\left(\widetilde{P}_{0}+\widetilde{P}_{1}\right)^{2} \gamma(z) \alpha_{1}(t) \\
& \leq \eta \frac{T \eta}{3}\left(\widetilde{P}_{0}+\widetilde{P}_{1}\right)^{2} \gamma(z) \alpha_{1}(t), \quad t \in[0, T] .
\end{aligned}
$$

Therefore, by virtue of (7.10), (7.5), we have

$$
\begin{aligned}
& \left|r_{3}(\beta(t), z)\right| \leq \eta\left(\widetilde{P}_{0}+\widetilde{P}_{1}\right)^{2} \gamma(z) \alpha_{2}(\beta(t)) \\
& \left.\leq \eta\left(\widetilde{P}_{0}+\widetilde{P}_{1}\right)^{2} \gamma(z) \frac{T}{3} \alpha_{1}(\beta(t))\right) \leq k_{T}(\beta) \frac{T \eta}{3}\left(\widetilde{P}_{0}+\widetilde{P}_{1}\right)^{2} \gamma(z) \alpha_{1}(t) \\
& \leq \eta \frac{T \eta}{3}\left(\widetilde{P}_{0}+\widetilde{P}_{1}\right)^{2} \gamma(z) \alpha_{1}(t), \quad t \in[0, T] .
\end{aligned}
$$


Estimates (6.9), (7.10), and (7.11) yield

$$
\begin{array}{r}
\left|r_{4}(t, z)\right| \leq \widetilde{P}_{0} \eta \frac{T \eta}{3}\left(\widetilde{P}_{0}+\widetilde{P}_{1}\right)^{2} \gamma(z) \alpha_{2}(t)+\widetilde{P}_{1} \eta \frac{T \eta}{3}\left(\widetilde{P}_{0}+\widetilde{P}_{1}\right)^{2} \gamma(z) \alpha_{2}(t) \\
=\eta \frac{T \eta}{3}\left(\widetilde{P}_{0}+\widetilde{P}_{1}\right)^{3} \gamma(z) \alpha_{2}(t) \leq\left(\frac{T \eta}{3}\right)^{2}\left(\widetilde{P}_{0}+\widetilde{P}_{1}\right)^{3} \gamma(z) \alpha_{1}(t) \\
\leq \eta\left(\frac{T \eta}{3}\right)^{2}\left(\widetilde{P}_{0}+\widetilde{P}_{1}\right)^{3} \gamma(z) \alpha_{1}(t), \quad t \in[0, T], \quad
\end{array}
$$

and, therefore, according to (7.12),

$$
\begin{aligned}
\left|r_{4}(\beta(t), z)\right| & \leq \eta \frac{\eta T}{3}\left(\widetilde{P}_{0}+\widetilde{P}_{1}\right)^{3} \gamma(z) \alpha_{2}(\beta(t)) \\
& \leq \eta \frac{\eta T}{3}\left(\widetilde{P}_{0}+\widetilde{P}_{1}\right)^{3} \gamma(z) \frac{T}{3} \alpha_{1}(\beta(t)) \\
& \leq \eta \frac{\eta T}{3}\left(\widetilde{P}_{0}+\widetilde{P}_{1}\right)^{3} \gamma(z) \frac{T}{3} k_{T}(\beta) \alpha_{1}(t) \\
& \leq \eta\left(\frac{\eta T}{3}\right)^{2}\left(\widetilde{P}_{0}+\widetilde{P}_{1}\right)^{3} \gamma(z) \alpha_{1}(t)
\end{aligned}
$$

for a. e. $t \in[0, T]$. Arguing by induction, we find that, for all $m \geq 1$, the estimates

$$
\begin{aligned}
\left|r_{m}(t, z)\right| & \leq\left(\frac{\eta T}{3}\right)^{m-2}\left(\widetilde{P}_{0}+\widetilde{P}_{1}\right)^{m-1} \gamma(z) \alpha_{1}(t) \\
& \leq\left(\widetilde{P}_{0}+\widetilde{P}_{1}\right) G_{2}^{m-2} \gamma(z) \alpha_{1}(t) \\
& \leq \eta\left(\widetilde{P}_{0}+\widetilde{P}_{1}\right) G_{2}^{m-2} \gamma(z) \alpha_{1}(t), \quad t \in[0, T],
\end{aligned}
$$

and

$$
\begin{aligned}
\left|r_{m}(\beta(t), z)\right| & \leq \eta\left(\frac{\eta T}{3}\right)^{m-2}\left(\widetilde{P}_{0}+\widetilde{P}_{1}\right)^{m-1} \gamma(z) \alpha_{1}(t) \\
& \leq \eta\left(\widetilde{P}_{0}+\widetilde{P}_{1}\right) G_{2}^{m-2} \gamma(z) \alpha_{1}(t), \quad t \in[0, T]
\end{aligned}
$$

are true, where

$$
G_{2}:=\frac{\eta T}{3}\left(\widetilde{P}_{0}+\widetilde{P}_{1}\right) .
$$


This, as in the proof of Theorem 1, yields

$$
\begin{aligned}
\left|x_{m+j}(t, z)-x_{m}(t, z)\right| & \leq \sum_{i=1}^{j}\left|r_{m+i}(t, z)\right| \\
& \leq \eta\left(\widetilde{P}_{0}+\widetilde{P}_{1}\right)\left(G_{2}^{m-1}+G_{2}^{m}+\cdots+G^{m+j-2}\right) \gamma(z) \alpha_{1}(t) \\
& =\eta\left(\widetilde{P}_{0}+\widetilde{P}_{1}\right) G_{2}^{m-1} \sum_{i=0}^{j-1} G_{2}^{i} \gamma(z) \alpha_{1}(t) \\
& \leq \eta\left(\widetilde{P}_{0}+\widetilde{P}_{1}\right) G_{2}^{m-1}\left(I-G_{2}\right)^{-1} \gamma(z) \alpha_{1}(t)
\end{aligned}
$$

for $t \in[0, T], m \geq 1, j \geq 1$. The last inequality proves that the members of sequence (4.1) satisfy estimates (4.4), where $G=G_{2}$ and the function $g:[0, T] \times \mathbb{R}^{n} \rightarrow \mathbb{R}^{n}$ is given by formula (7.7). By virtue of condition (7.6) and Definition 1, this means that method (4.1) for problem (3.1), (3.2) is applicable with an estimate of the type $\left(T \eta\left(\widetilde{P}_{0}+\widetilde{P}_{1}\right) / 3, g\right)$, as required.

Remark 3. In the cases where the value $k_{T}(\beta)$ in the assumption (5.10) of Lemma 4 satisfies the inequality

$$
1 \leq k_{T}(\beta)<\frac{3}{2},
$$

condition (7.6) of Theorem 3 is less restrictive than condition (6.1) of Theorem 1.

\section{EXAMPLES}

The conditions of the theorems presented above are easily verified in concrete cases.

Example 2. Let us consider the system of two differential equations with argument deviation

$$
\begin{aligned}
& x_{1}^{\prime}(t)=\frac{t}{2} x_{1}\left(t^{2}\right)+x_{2}(t)-\frac{t}{8}\left(t^{4}+1\right), \\
& x_{2}^{\prime}(t)=\frac{1}{2} x_{1}(t)-\frac{1}{2} x_{2}\left(t^{2}\right)+\frac{3}{8}+\frac{t^{2}}{8}, \quad t \in[0,1],
\end{aligned}
$$

under the inhomogeneous two-point boundary conditions

$$
\begin{gathered}
\frac{1}{2} x_{1}(0)+x_{2}(0)-\frac{1}{4} x_{2}(1)=0, \\
x_{1}(0)+x_{1}(1)-x_{2}(1)=\frac{1}{4} .
\end{gathered}
$$

One can verify directly that the pair of functions

$$
x_{1}(t)=\frac{1}{4}\left(t^{2}+1\right), \quad x_{2}(t)=\frac{t}{2}, \quad t \in[0,1],
$$

is a solution of problem (8.1), (8.2), (8.3). 
Problem (8.1), (8.2), (8.3) is, of course, a particular case of problem (3.1), (3.2) when $T=1$,

$$
f_{1}(t)=-\frac{t}{8}\left(t^{4}+1\right), \quad f_{2}(t)=\frac{3}{8}+\frac{t^{2}}{8}, \quad t \in[0,1],
$$

the function determining the deviation of argument has the form

$$
\beta(t)=t^{2}, \quad t \in[0,1],
$$

the matrix-valued functions $P_{0}$ and $P_{1}$ are given by the equalities

$$
P_{0}(t)=\left(\begin{array}{cc}
0 & 1 \\
\frac{1}{2} & 0
\end{array}\right), \quad P_{1}(t)=\left(\begin{array}{cc}
\frac{t}{2} & 0 \\
0 & -\frac{1}{2}
\end{array}\right), \quad t \in[0,1],
$$

and $f(t)=\operatorname{col}\left(f_{1}(t), f_{2}(t)\right), t \in[0,1]$. It is also clear that the boundary conditions (8.3), (8.4) can be rewritten in form (3.2) with

$$
A=\left(\begin{array}{cc}
\frac{1}{2} & 1 \\
1 & 0
\end{array}\right), \quad B=\left(\begin{array}{cc}
0 & -\frac{1}{4} \\
1 & -1
\end{array}\right), \quad d=\left(\begin{array}{c}
0 \\
\frac{1}{4}
\end{array}\right) .
$$

The argument deviation (8.6) satisfies (5.10), and, therefore, by Lemma 4, the value $k_{T}(\beta)$ is finite and estimate (5.11) is true. It is easy to see that, in this case, $k_{T}(\beta)=2$ because, in view of (5.2) and (8.6), $\alpha_{1}(\beta(t))\left(\alpha_{1}(t)\right)^{-1}=2 t^{2}\left(1-t^{2}\right)\left(\alpha_{1}(t)\right)^{-1}=$ $t(1+t)$ for $t \in[0,1]$.

It is obvious from (6.2) and (8.7) that, in this case,

$$
\widetilde{P}_{0}=\left(\begin{array}{cc}
0 & 1 \\
\frac{1}{2} & 0
\end{array}\right), \quad \widetilde{P}_{1}=\left(\begin{array}{cc}
\frac{1}{2} & 0 \\
0 & \frac{1}{2}
\end{array}\right) .
$$

Therefore, condition (7.6) is satisfied because the greatest eigenvalue of the matrix $\widetilde{P}_{0}+\widetilde{P}_{1}=\left(\begin{array}{cc}\frac{1}{2} & 1 \\ \frac{1}{2} & \frac{1}{2}\end{array}\right)$ is equal to $(1+\sqrt{2}) / 2 \approx 1.207$, and, hence, is less than $3 T^{-1} \max \left\{1, k_{T}(\beta)\right\}=\frac{3}{2}$. By virtue of Theorem 3, the method of successive approximations (4.1) is applicable for problem (8.1), (8.2), (8.3) with an estimate of the type

$$
\left(\begin{array}{ll}
\frac{2}{3} & \left.\begin{array}{cc}
\frac{1}{2} & 1 \\
\frac{1}{2} & \frac{1}{2}
\end{array}\right), 3 \alpha_{1} \gamma
\end{array}\right),
$$

where $\alpha_{1}$ and $\gamma$ are functions given by formulae (5.2) and (6.3), respectively. Therefore, one can use Theorem 3 and construct the successive approximations defined by formula (4.1). After solving the approximate determining equations (4.14) for some $m$, this, according to Remark 1, will allow one to obtain approximations (4.15) to the solutions in question. 
Let us put $x_{0 i}(t):=z_{i}, i=1,2, t \in[0,1]$, and construct the corresponding functions $x_{m}=\left(\begin{array}{c}x_{m 1} \\ x_{m 2}\end{array}\right):[0,1] \times \mathbb{R} \rightarrow \mathbb{R}^{2}$ for some $m$. For $m=1$, formula (4.1) gives

$$
\begin{aligned}
& x_{11}(t, z)=-\frac{t^{6}}{48}+\frac{1}{4}\left(z_{1}-\frac{1}{4}\right) t^{2}+\left(4 z_{2}-\frac{z_{1}}{4}+\frac{1}{3}\right) t+z_{1}, \\
& x_{12}(t, z)=\frac{t^{3}}{24}+\left(2 z_{1}+3 z_{2}-\frac{1}{24}\right) t+z_{2}, \quad t \in[0,1],
\end{aligned}
$$

and the corresponding equation (4.14) has the root

$$
z_{1} \approx 0.2541165999, \quad z_{2} \approx 0.006962987687
$$

Substituting the values (8.12) into formulae (8.10) and (8.11), we obtain the first approximation (4.15) of the form

$$
\begin{aligned}
& X_{11}(t)=0.2541165999-\frac{t^{6}}{48}+0.001029150000 t^{2}+0.2976561342 t \\
& X_{12}(t)=0.006962987687+0.4874554962 t+\frac{t^{3}}{24}, \quad t \in[0,1] .
\end{aligned}
$$

We see from Figure 2 that the function $X_{11}$ still differs significantly from the first component of the exact solution (8.5).
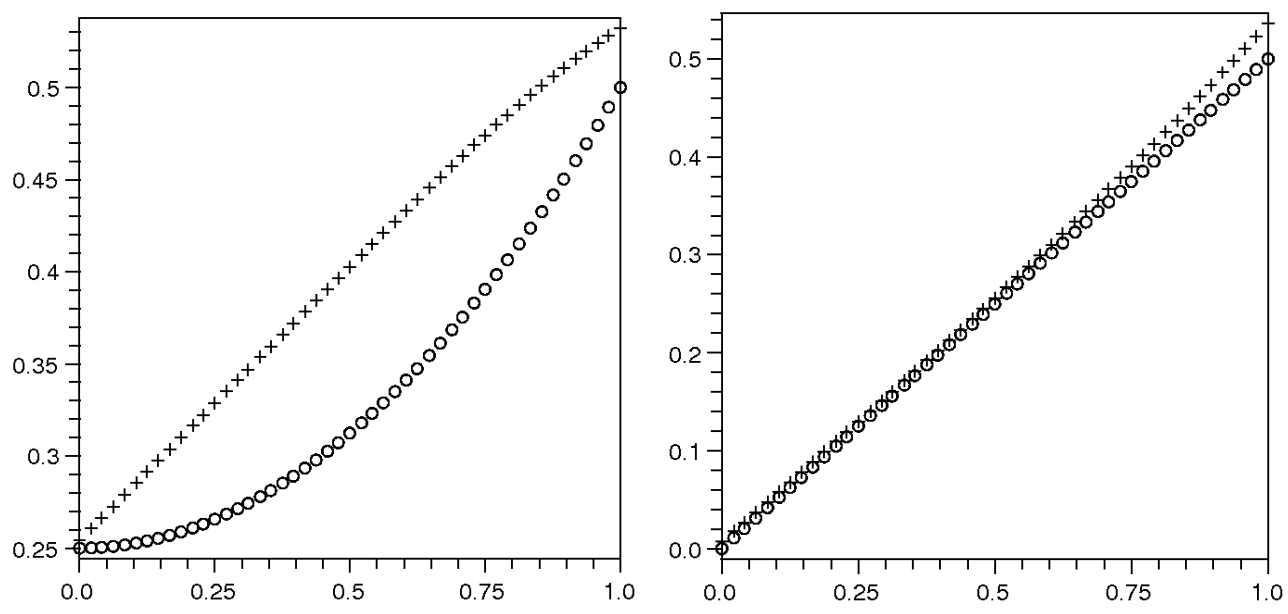

FIGURE 2. The first approximation (crosses) and the exact solution (circles) of the boundary value problem (8.1), (8.2), (8.3), (8.4) 
Using expressions (8.10) and (8.11) for $x_{1 i}, i=1,2$, and computing function (4.1) for $m=2$, we obtain

$$
\begin{aligned}
x_{21}(t, z) & =-\frac{t^{14}}{1344}+\frac{1}{48}\left(z_{1}-\frac{5}{4}\right) t^{6}+\frac{1}{32}\left(\frac{5}{3}+16 z_{2}-z_{1}\right) \\
& +\frac{1}{4}\left(6 z_{2}-\frac{1}{3}+5 z_{1}\right) t^{2}+\frac{1}{32}\left(64 z_{2}+\frac{69}{7}-\frac{119 z_{1}}{3}\right) t, \\
x_{22}(t, z) & =-\frac{1}{224} t^{7}+\frac{1}{2}\left(\frac{11}{144}-\frac{7 z_{1}}{12}-z_{2}\right) t^{3}+\left(z_{2}+\frac{1}{12}-\frac{z_{1}}{16}\right) t^{2} \\
& +\frac{1}{2}\left(\frac{113 z_{1}}{24}+5 z_{2}-\frac{59}{252}\right) t+z_{2}, \quad t \in[0,1],
\end{aligned}
$$

and the corresponding system of approximate determining equations (4.14) has the root

$$
z_{1} \approx 0.2506508168, \quad z_{2} \approx-0.0005320453803 \text {. }
$$

Substituting (8.15) into (8.13) and (8.14), we obtain the second approximation, the graphs of whose components are presented on Figure 3:

$$
\begin{aligned}
X_{21}(t)= & 0.2506508168-\frac{t^{14}}{1344}-0.02081977465 t^{6}+0.04398447260 t^{4} \\
& +0.2291821196 t^{2}, \\
X_{22}(t)= & -0.0005320453803-\frac{t^{7}}{224}-0.03464602110 t^{3}+0.06713561190 t^{2} \\
& +0.4716801924 t, \quad t \in[0,1] .
\end{aligned}
$$
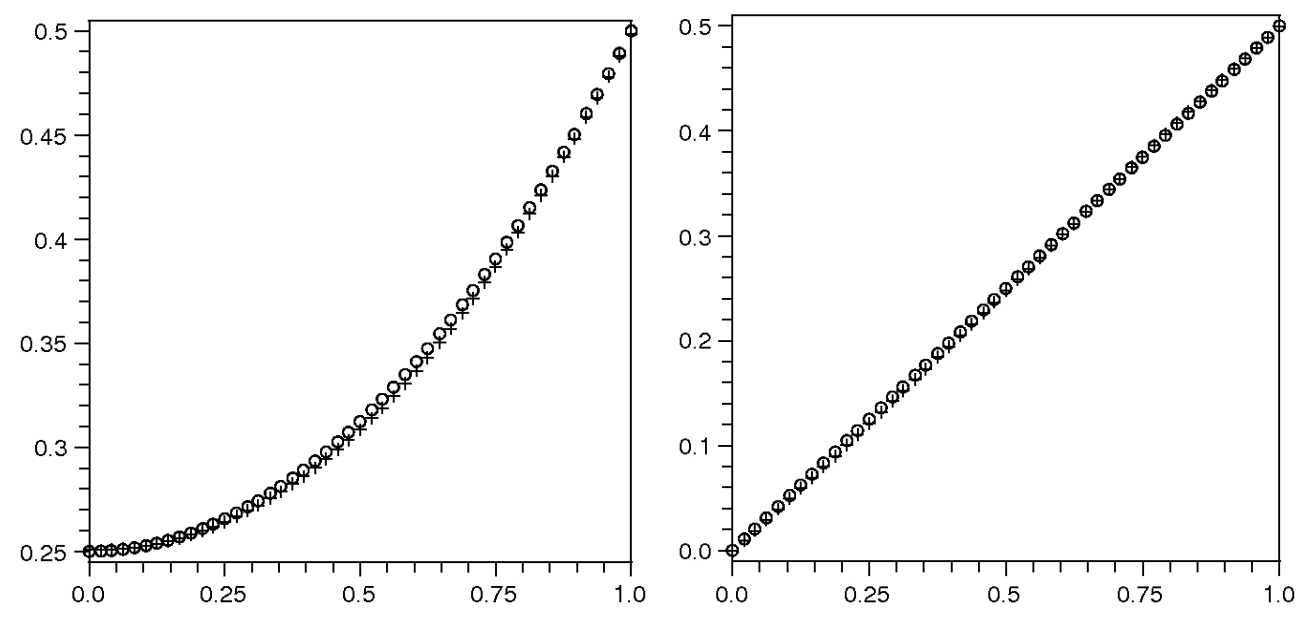

FIGURE 3. The second approximation (crosses) and the exact solution (circles) of the boundary value problem (8.1), (8.2), (8.3), (8.4) 
Higher approximations are constructed by analogy. For example, using Maple,one obtains the roots of the fourth approximate determining equation

$$
z_{1} \approx 0.2499876647, \quad z_{2} \approx 0.000007202426452
$$

and the corresponding fourth approximation

$$
\begin{aligned}
X_{41}(t) & =0.2499876647-\frac{t^{62}}{9999360}-0.00001240094662 t^{30} \\
& +0.00005031229545 t^{22}-\frac{t^{18}}{64512}+\frac{t^{16}}{129024}-0.00006201770500 t^{14} \\
& -0.0004454679680 t^{10}+0.001534762062 t^{8}-0.001579817533 t^{6} \\
& +0.0005047247500 t^{4}-0.0001525352967 t^{3}+0.2501969927 t^{2} \\
& +0.0000021079 t \\
X_{42}(t) & =0.000007202426452-\frac{11 t^{31}}{4999680}-0.00005787007260 t^{15} \\
& +0.0002071768032 t^{11}-\frac{t^{9}}{32256}-0.0002156414800 t^{7} \\
& -0.0008451753460 t^{5}+0.002821512780 t^{4}-0.002444944367 t^{3} \\
& +0.0003682070000 t^{2}+0.5001968735 t, \quad t \in[0,1]
\end{aligned}
$$

The results of computation clearly show that functions (8.19) and (8.20) provide a good approximation of solution (8.5). In particular, numbers (8.18) are rather accurate approximations of the initial values $\frac{1}{4}$ and 0 of the exact solution at the point 0 (see also Table 1). In fact, already starting from $m=2$, the graph of the $m$ th approximation constructed according to the method under consideration practically coincides with that of the exact solution.

\begin{tabular}{|c|c|c|}
\hline$i$ & $X_{i 1}(0)$ & $X_{i 2}(0)$ \\
\hline \hline 1 & 0.2541165999 & 0.006962987687 \\
2 & 0.2506508168 & -0.0005320453803 \\
3 & 0.2499480368 & -0.00003329847918 \\
4 & 0.2499876647 & 0.000007202426452 \\
\hline
\end{tabular}

TABLE 1. Initial values of the approximate solutions of problem (8.1), (8.2), (8.3)

Along with condition (7.6), inequality (6.1) for the problem considered in Example 2 is satisfied as well. It should be noted, however, that condition (3.6), which follows from the assumptions of the work [1], is not fulfilled. 

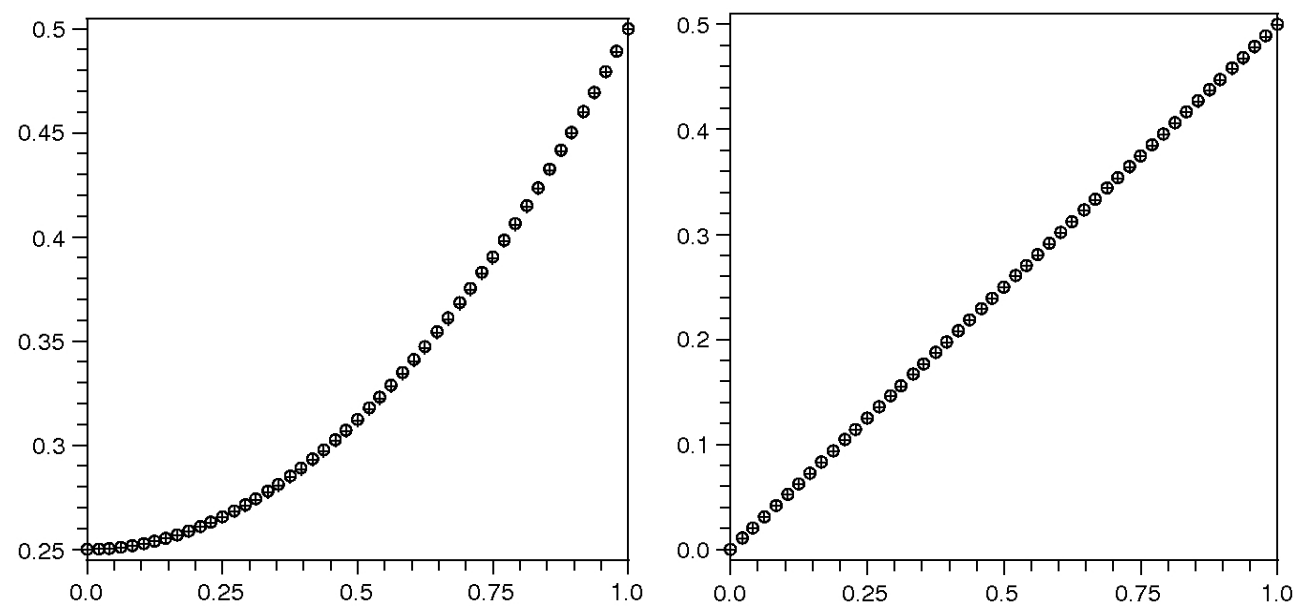

FIGURE 4. The third approximation (crosses) and the exact solution (circles) of the boundary value problem (8.1), (8.2), (8.3), (8.4)

Example 3. Consider the boundary value problem (8.3), (8.4) for the system of equations

$$
\begin{aligned}
& x_{1}^{\prime}(t)=\frac{t}{2} x_{1}\left(1-\frac{3 t}{4}(1-t)\right)+x_{2}(t)-\frac{t}{8}\left(1+\left(1-\frac{3 t}{4}(1-t)\right)^{2}\right), \\
& x_{2}^{\prime}(t)=\frac{1}{2} x_{1}(t)-\frac{1}{2} x_{2}\left(1-\frac{3 t}{4}(1-t)\right)+\frac{1}{16}\left(10-3 t+t^{2}\right), \quad t \in[0,1],
\end{aligned}
$$

Clearly, problem (8.21), (8.22), (8.3) is a particular case of problem (3.1), (3.2), where $T=1$,

$f_{1}(t)=-\frac{t}{8}\left(1+\left(1-\frac{3 t}{4}(1-t)\right)^{2}\right), \quad f_{2}(t)=\frac{1}{16}\left(10-3 t+t^{2}\right), \quad t \in[0,1]$,

the argument deviation $\beta$ has the form

$$
\beta(t)=1-\frac{3 t}{4}(1-t), \quad t \in[0,1],
$$

and $P_{0}, P_{1}, A, B$, and $d$ are given by formulae (8.7), (8.8). Function (8.23), as is easy to verify, transforms the interval $[0,1]$ into itself and satisfies condition (5.10), and the value of $k_{T}(\beta)$ in (5.11) is equal to $\frac{3}{4}$. Therefore, by Theorem 3, the method of successive approximations for problem (8.21), (8.22), (8.3) is applicable with an estimate of the type

$$
\left(\frac{1}{3}\left(\begin{array}{cc}
\frac{1}{2} & 1 \\
\frac{1}{2} & \frac{1}{2}
\end{array}\right), 3 \alpha_{1} \gamma\right)
$$


where $\alpha_{1}$ and $\gamma$ are functions given by formulae (5.2) and (6.3), respectively. It is worth pointing out that estimate (8.24) is sharper than estimate (8.9) appearing in Example 2, which differs from Example 3 only by the forcing terms and the type of argument deviation. Note that the same function (8.5) is an exact solution of the problem indicated.

\begin{tabular}{|c|c|c|}
\hline$i$ & $X_{i 1}(0)$ & $X_{i 2}(0)$ \\
\hline \hline 0 & 0.5429142442 & -0.07493943798 \\
1 & 0.2407327110 & 0.01169161945 \\
2 & 0.2497717670 & -0.0001512180660 \\
3 & 0.2500928841 & -0.00009129853295 \\
4 & 0.2500037453 & 0.000002253878265 \\
5 & 0.2499986308 & 0.000001057944723 \\
\hline
\end{tabular}

TABLE 2. Initial values of the approximate solutions of problem (8.21), (8.22), (8.3)

Carrying out the computation according to formulae (4.1) and solving the approximate determining equations (4.14), we have constructed five approximations $X_{i 1}$, $X_{i 2}, i=1, \ldots, 5$, to the respective components of a solution of problem (8.21), (8.22), (8.3). The results of computation (see, e. g., Table 2) show rather clearly that the approximations constructed according the scheme suggested are very accurate already on the first few steps of iteration.

\section{ACKNOWLEDGEMENTS}

The research was supported in part by the Academy of Sciences of the Czech Republic, Institutional Research Plan No. AV0Z10190503, Grant Agency of the Czech Republic through Grant No. 201/06/0254 (A. Rontó), and the Hungarian Scientific Research Fund OTKA through Grant No. K68311 (M. Rontó).

\section{REFERENCES}

[1] A. Augustynowicz and M. Kwapisz, "On a numerical-analytic method of solving of boundary value problem for functional-differential equation of neutral type," Math. Nachr., vol. 145, pp. 255-269, 1990.

[2] R. E. Gaines and J. L. Mawhin, Coincidence degree, and nonlinear differential equations., ser. Lecture Notes in Mathematics 568. Berlin-Heidelberg-New York: Springer-Verlag, 1977.

[3] M. A. Krasnoselskii, G. M. Vainikko, P. P. Zabreiko, Y. B. Rutitskii, and V. Y. Stetsenko, Approximate Solution of Operator Equations. Groningen: Wolters-Noordhoff Scientific Publications Ltd., 1972.

[4] M. Kwapisz, "On modification of the integral equation of A. M. Samoilenko's numerical-analytic method," Math. Nachr., vol. 157, pp. 125-135, 1992. 
[5] M. Kwapisz, "Some remarks on an integral equation arising in applications of numerical-analytic method of solving of boundary value problems," Ukrain. Math. J., vol. 44, no. 1, pp. 115-119, 1992.

[6] M. Kwapisz, "On integral equations arising in numerical-analytic method of solving boundary value problems for differential-functional equations," in International Conference on Differential Equations, Vol. 1, 2 (Barcelona, 1991). World Sci. Publ., River Edge, NJ, 1993, pp. 671-677.

[7] A. Ronto and M. Rontó, "A note on the numerical-analytic method for nonlinear two-point boundary-value problems," Nonlinear Oscil., vol. 4, no. 1, pp. 112-128, 2001.

[8] A. Ronto, "On some boundary value problems for the Lipschitz differential equations." Nelinijni Kolyvannya, vol. 1998, no. 1, pp. 74-94, 1998.

[9] M. Rontó and J. Mészáros, "Some remarks on the convergence analysis of the numerical-analytic method based on successive approximations," Ukrainian Math. J., vol. 48, no. 1, pp. 101-107, 1996.

[10] M. Ronto and A. Samoilenko, Numerical-Analytic Methods in the Theory of Boundary-Value Problems. River Edge, NJ: World Scientific Publishing Co. Inc., 2000, with a preface by Yu. A. Mitropolsky and an appendix by the authors and S. I. Trofimchuk.

[11] N. I. Ronto, A. M. Samoilenko, and S. I. Trofimchuk, "The theory of the numerical-analytic method: achievements and new directions of development. I," Ukrainian Math. J., vol. 50, no. 1, pp. 116-135, 1998.

[12] N. I. Ronto, A. M. Samoilenko, and S. I. Trofimchuk, "The theory of the numerical-analytic method: achievements and new directions of development. II," Ukrainian Math. J., vol. 50, no. 2, pp. 255-277, 1998.

[13] N. I. Ronto, A. M. Samoilenko, and S. I. Trofimchuk, "The theory of the numerical-analytic method: achievements and new directions of development. III," Ukrainian Math. J., vol. 50, no. 7 , pp. 1091-1114, 1998.

[14] N. I. Ronto, A. M. Samoilenko, and S. I. Trofimchuk, "The theory of the numerical-analytic method: achievements and new directions of development. IV," Ukrainian Math. J., vol. 50, no. 12, pp. 1888-1907, 1998.

[15] N. I. Ronto, A. M. Samoilenko, and S. I. Trofimchuk, "The theory of the numerical-analytic method: achievements and new directions of development. V," Ukrainian Math. J., vol. 51, no. 5, pp. 735-747, 1999.

[16] N. I. Ronto, A. M. Samoilenko, and S. I. Trofimchuk, "The theory of the numerical-analytic method: achievements and new directions of development. VI," Ukrainian Math. J., vol. 51, no. 7, pp. 1079-1094, 1999.

[17] N. I. Ronto, A. M. Samoilenko, and S. I. Trofimchuk, "The theory of the numerical-analytic method: achievements and new directions of development. VII," Ukrainian Math. J., vol. 51, no. 9, pp. 1399-1418, 1999.

[18] N. Rouche and J. Mawhin, Ordinary differential equations, ser. Surveys and Reference Works in Mathematics. Boston, Mass.: Pitman (Advanced Publishing Program), 1980, vol. 5, stability and periodic solutions, Translated from the French and with a preface by R. E. Gaines.

[19] A. M. Samoilenko, "A numerical-analytic method for the investigation of periodic systems of ordinary differential equations. I," Ukr. Mat. Zh., vol. 17, no. 4, pp. 82-93, 1965.

[20] A. M. Samoilenko, "A numerical-analytic method for the investigation of periodic systems of ordinary differential equations. II," Ukr. Mat. Zh., vol. 18, no. 2, pp. 50-59, 1966.

[21] A. M. Samoilenko and N. I. Ronto, Numerical-analytic methods of investigation of periodic solutions. Kiev: Višča Škola, 1976.

[22] A. M. Samoilenko and N. I. Ronto, Numerical-analytic methods of investigating periodic solutions. Moscow: Mir, 1979, translated from the Russian by Vladimir Shokurov, With a foreword by Yu. A. Mitropol'skiı̌. 
[23] A. M. Samoilenko and N. I. Ronto, Numerical-analytic methods in the theory of boundary value problems for ordinary differential equations. Kiev: Naukova Dumka, 1992.

Authors' addresses

A. Rontó

Institute of Mathematics, Academy of Sciences of the Czech Republic, Žižkova 22, CZ-61662, Brno,

Czech Republic

E-mail address: ronto@math.cas.cz

M. Rontó

Department of Analysis, University of Miskolc, 3515 Miskolc-Egyetemváros, Hungary

E-mail address: matronto@gold.uni-miskolc.hu 